\title{
Differential phase tracking applied to Bragg gratings in multicore fiber for high accuracy curvature measurement
}

\author{
Gordon M. H. Flockhart ${ }^{*}$, Geoffrey A. Cranch ${ }^{\mathrm{a}}$, Clay K. Kirkendall ${ }^{\mathrm{b}}$ \\ ${ }^{a}$ SFA Inc., 2200 Defense Highway, Suite 405, Crofton, MD 21114, USA; \\ ${ }^{\mathrm{b}}$ Naval Research Laboratory, 4555 Overlook Ave. SW, Washington, DC 20375, USA
}

\begin{abstract}
We report interferometric interrogation of fiber Bragg gratings in separate cores of a multicore fiber for high resolution quasi-static and dynamic bend measurements. Two axis curvature measurements are made by measuring the differential strain between three FBG sensors formed in a singlemode four-core fiber using a common interrogating interferometer. Therefore a measurement of the differential phase from each FBG yields the differential strain and compensates for the common-mode random drift of the interrogating interferometer. A DC curvature accuracy of $3.4 \times 10^{-3} \mathrm{~m}^{-1}$, and an AC curvature resolution of $1.2 \times 10^{-4} \mathrm{~m}^{-1} / \mathrm{Hz}^{1 / 2}$ are reported.
\end{abstract}

Keywords: Optical fiber sensor, fiber Bragg grating, interferometry, curvature measurement, multicore

\section{INTRODUCTION}

The term 'smart structure' is commonly applied to structures with embedded or adhered sensors, which allow the mechanical state of the structure to be determined. For example, structures with sensors within them could continuously monitor their internal strain, temperature, vibration, and structural integrity. A wide range of information could be obtained about the structure and used to evaluate the loads exerted on the structure, the changes in the structure's environment, or to provide suitable information for repair and servicing. This information is important for improving the design of structures based on new materials, such as composite materials, or for long term monitoring, providing a continuous record of the state of the structure.

The sensing systems could also be developed as feedback or control mechanisms for advanced smart structures incorporating actuators where the information provided by the sensors is used to control or modify some aspect of the structure, such as its shape or orientation. The possible applications for adaptive smart structures are vast. In future generations of active smart structures, sensing systems could provide information to neural network systems allowing the structure to learn and adapt to its environment ${ }^{1}$.

One of the main uses of sensors in smart structures is to determine the strain throughout the structure and this can be measured relatively easily using electrical strain gauges, which have been an established engineers' tool since the 1940 's ${ }^{2}$. Smart structures are often made of composite materials using carbon, glass or Kevlar fibers and although electrical strain gauges can be embedded in these materials, the electrical wiring associated with them complicates the process. Electrical strain gauges are not readily multiplexed and for applications where measurements of large numbers of strain gauges are required the electrical wiring can be a significant problem.

Optical fiber strain gauges have many potential advantages over electrical gauges for the use in smart structures. The small physical size of the optical fiber is more suitable to being embedded within the composite structure during its fabrication. The material properties of optical fibers are also attractive; the material strength is quite high, in particular the tensile strength and they are highly resistant to fatigue as the silica exhibits excellent elasticity up to its breaking point. Optical fiber Bragg grating (FBG) strain gauges can be readily multiplexed onto a single fiber using techniques such as time division multiplexing or wavelength division multiplexing ${ }^{3}$. FBG strain gauges have been used for structural monitoring in civil, marine, and aerospace applications ${ }^{4}$. Strain measurements within structures are often used to determine structural deformation; bending is inferred from the measured $\operatorname{strain}^{5}$. Curvature or bending in structures *E-mail: gordon.flockhart@nrl.navy.mil; Phone: +1 202767 9526; Fax: +1 202767 5792; www.nrl.navy.mil

Smart Structures and Materials 2006: Smart Sensor Monitoring Systems and Applications, edited by Daniele Inaudi, Wolfgang Ecke, Brian Culshaw, Kara Peters, and Eric Udd, Proc. of SPIE Vol. 6167, 616701, (2006) · 0277-786X/06/\$15 doi: $10.1117 / 12.657576$

Proc. of SPIE Vol. 6167 616701-1 
can be calculated by measuring the bending induced strain in the structure at a fixed and known distance from the neutral axis of the structure. The accuracy of the bend measurement is, therefore, dependent on the strain transfer between the structure and FBG strain gauges, and on precise knowledge of the of the gauge locations. Curvature sensors using FBG strain gauges have been reported by measuring differential strain in a structure ${ }^{6}$. To measure one-axis curvature two independent strain gauges are separated by a fixed distance $d$ in a structure. When the structure is bent with radius of curvature $R$, in a plane containing the two strain gauges, the strain difference between the cores is given by $\varepsilon_{1}-\varepsilon_{2}=d / R$. Temperature-insensitive curvature measurement has been demonstrated by applying FBG strain gauges to opposite sides of a cantilever beam ${ }^{6}$. This measurement is only insensitive to temperature when the cantilever beam is in thermal equilibrium and hence the two FBGs are at the same temperature. However due to the temperature cross sensitivity of FBG strain gauges, a temperature gradient across the beam would give rise to an apparent strain difference between the FBGs and thus a temperature induced curvature error.

Sensors that can directly measure curvature are desirable for a number of reasons. In applications where the sensor is embedded within the structure, the precise location of the sensor need not be known in order to determine the curvature. This is important for composite materials in which the sensor location may change during the cure process. Curvature sensors can also be located along the neutral axis of the structure where the bending strain is zero and hence is not a suitable location for strain gauges. A direct measurement of curvature is increasingly important for thin structures where the bending strain is considerably reduced.

A number of optical fiber curvature sensors have been proposed based on a range of different sensing technologies. These include attenuation based one-axis sensors, where the optical fiber has been specially treated to introduce transmission loss which is dependent on the magnitude and bend direction ${ }^{7,8}$. Curvature sensors based on the wavelength and attenuation changes in long-period gratings ${ }^{9}(\mathrm{LPG})$, and resonance mode splitting of $\mathrm{LPGs}^{10,11}$ have been demonstrated. In optical fibers with perfect core concentricity and circular claddings these sensors would not exhibit any directional sensitivity; however errors in the core concentricity can give rise to asymmetric responses. Improved directional curvature sensors have been demonstrated in eccentric core fibers ${ }^{12}$, D-shaped fibers ${ }^{13}{ }^{14}$, flat-clad fibers ${ }^{15}$ and multicore fibers ${ }^{15}$. More recently, femtosecond laser processing was used to produce an asymmetric refractive index change in the fiber's cladding to produce a directional LPG curvature sensor ${ }^{16}$. Typically LPG curvature sensors are limited to one-axis sensing and are also cross sensitive to temperature and strain, but the temperature sensitivity can be reduced ${ }^{17}$ or it is possible to perform simultaneous bend and temperature sensing ${ }^{10}$. Two-axis bend sensing could be achieved by co-locating two directional curvature sensors. Recently a two-axis bend sensor was reported using multiple LPGs fabricated using a $\mathrm{CO}_{2}$ laser ${ }^{18}$. However, for all of these different curvature sensors, the number of sensors that can be multiplexed along a single fiber is limited. Multiple sensors are required for two-axis bend measurement on extended structures, such as long hydrophone arrays.

As mentioned previously, many FBG sensors can be multiplexed along a single fiber; however FBG sensors are not directly sensitive to curvature but can be used to measure differential strain. Ideally, it is desirable to place the FBG strain gauges as close together as possible in order to minimize the sensitivity to thermal gradients; however doing so also reduces differential strain for a given curvature. A miniature one-axis curvature sensor has been reported by bonding two FBGs in D-shaped fiber back to back ${ }^{19}$. By measuring the differential strain between the two FBGs the curvature can determined independently of common mode signals such as longitudinal strain and temperature. Taking this idea one step further, two-axis curvature measurement using FBGs has been demonstrated by stacking three single-mode fibers with FBG strain gauges in a triangular configuration ${ }^{20}$. This allows determination of the curvature size and direction independently of strain and temperature. However, the long-term stability and accuracy of these sensors depends on the bonding and strain transfer between the separate optical fibers. A more robust curvature sensor can be fabricated by writing FBG strain gauges into three cores of a multicore fiber $(\mathrm{MCF})^{21}$. Having all three FBG strain gauges in the same host structure greatly improves the mechanical and thermal stability of the sensor. The MCF geometry accurately defines the core separation and promotes good thermal stability between cores as a result of the small core separation. However, the small core separation requires improved strain measurement. High-resolution and dynamic range strain measurement can be achieved with an interferometric wavelength discriminator. This yields strain resolutions approaching the nanostrain level over large bandwidths. Interferometric interrogation of fiber Bragg grating strain sensors has demonstrated resolutions of $6 \mathrm{n} \varepsilon / \mathrm{Hz}^{1 / 2}$ at $1 \mathrm{~Hz}$ for a single sensor ${ }^{22}$ and $4 \mathrm{n} \varepsilon / \mathrm{Hz}^{1 / 2}$ at $0.1 \mathrm{~Hz}$ for a multiplexed array ${ }^{23}$. 
In this paper we report the application of interferometric interrogation for differential strain sensing in separate cores of a MCF for high resolution quasi-static and dynamic curvature measurements.

\section{GRATING FABRICATION IN MULTICORE FIBER}

The MCF consists of four Ge-doped cores arranged in a square with a core separation of $\sim 50 \mu \mathrm{m}$ and a circular cladding diameter of $125 \mu \mathrm{m}$ [Fig. 1 (a)]. The four cores are singlemode at $1550 \mathrm{~nm}$ and the Ge-dopant concentration was chosen to increase the fiber's photosensitivity for the purpose of grating fabrication. Fiber Bragg gratings were fabricated in all four cores of the MCF using a continuous wave frequency-doubled argon-ion laser ( $\lambda=244 \mathrm{~nm}$ and $\mathrm{P}_{\text {output }}=125 \mathrm{~mW}$ ) and uniform period phase mask. The laser beam is focused using a cylindrical lens to a line with a $1 / \mathrm{e}^{2}$ width of $\sim 26 \mu \mathrm{m}$; a slit aperture truncates the beam to a length of $12 \mathrm{~mm}$. In order to fabricate similar gratings in all four cores the orientation of the MCF is investigated by attenuating and scanning the writing beam perpendicular to the fiber axis. The total fluorescence irradiance coupled into the fiber cores is recorded as a function of position and is used to determine the fiber orientation. The narrow beam waist of the writing beam allows the cores to be identified and Fig. 1 (b) shows the recorded fluorescence profiles for three different fiber orientations. If we consider the writing beam to be incident on the image of the MCF [Fig. 1 (a)] from the right hand side, fluorescence profile number 1 indicates the fiber is rotated $\sim 45^{\circ}$ from what is shown; whereas profile number 3 indicates the geometry shown in Fig. 1 (a). Two separate exposures were used to fabricate the FBGs. The writing beam was first aligned to the top two cores and a $5 \mathrm{sec}$ exposure resulted in the fabrication of gratings predominantly in cores 1 and 4 . The beam was then aligned to bottom two cores and a second 5 sec exposure produced gratings in cores 2 and 3. The reflection spectra of the FBGs are shown in Fig. 1 (c). It can be seen that the Bragg wavelengths are different in each core, ranging from $1550.53 \mathrm{~nm}$ to $1551.77 \mathrm{~nm}$. The grating strengths also vary from core to core, ranging from $1-4 \mathrm{~dB}$. The difference in grating strength can be attributed to variations in the UV irradiance incident on each core due to the focusing effect of the optical fiber; however the spread in the Bragg wavelengths is too large to be solely due to different exposure conditions. We believe the different Bragg wavelengths are due to variations in the effective refractive index of each core.

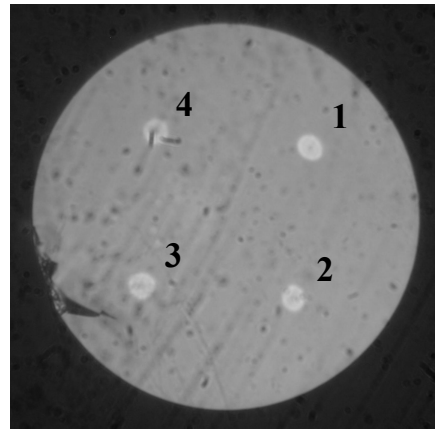

(a)

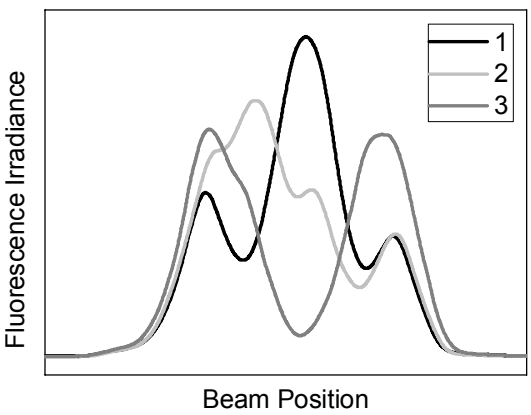

(b)

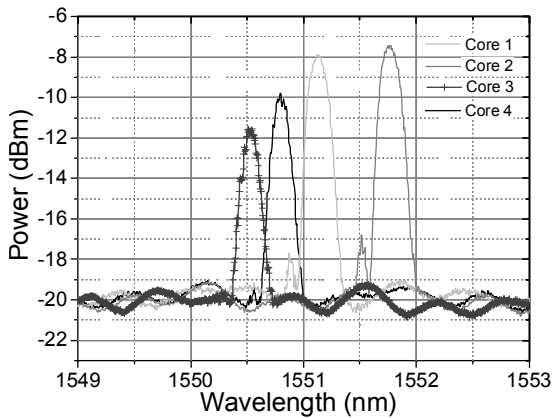

(c)

Fig. 1. (a) Cleaved face of MCF; (b) fluorescence profiles for three azimuthal orientations of the MCF and (c) unstrained FBG reflection spectra in individual cores of $\mathrm{MCF}$.

\section{EXPERIMENTAL SET-UP}

\subsection{Bending test-rig}

Fig. 2 is a schematic of the experiment to apply bending and measure the induced differential strain. The bending test rig comprised a cantilever beam formed by a brass tube of outer diameter $1.6 \mathrm{~mm}$. The FBGs in the MCF were recoated using a $410 \mu \mathrm{m}$ mold and inserted into a PTFE tube which was then inserted into the brass cantilever beam. The PTFE tubing was used to ensure a well toleranced fit in the brass tube; thus ensuring the fiber took the shape of the cantilever beam when deflected. The FBGs were located $3.5 \pm 1 \mathrm{~mm}$ from the fixed end of the cantilever beam. The cantilever was displaced by two orthogonal motorized micrometers at a distance of $199 \mathrm{~mm}$ from the fixed end. This configuration allows small curvatures to be applied independently along two axes; however this arrangement does not produce constant strain along the length of the fiber. For small deflections of the cantilever beam, the curvature at a distance $z$ from the fixed end of the cantilever is given by 


$$
\frac{1}{R}=\kappa=\frac{3(l-z)}{l^{3}} v_{l}
$$

for displacement $v_{l}$ at loading point $l$ from the fixed end of the cantilever ${ }^{24}$. There will be a variation in strain along the length of the FBG which will induce a chirp in the grating period. However this effect will be of equal magnitude but of opposite sign for the FBGs in tension versus compression.

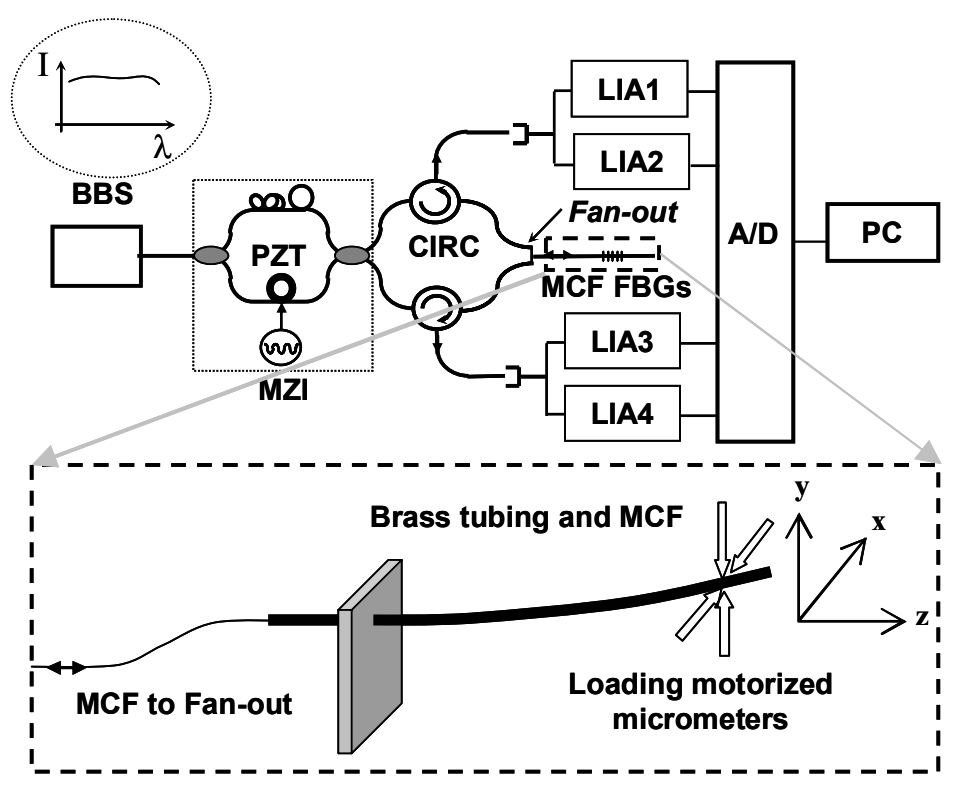

Fig. 2. Experimental set-up for curvature measurement

\subsection{Mach-Zehnder interferometric demodulator}

The curvature induced differential strain was measured using an interferometric demodulation technique. The output from an erbium broadband source (BBS) is launched into a fiber-optic Mach-Zehnder interferometer (MZI) with a 3.6 $\mathrm{mm}$ optical path imbalance, which is smaller than the coherence length of the light reflected from a sensor grating. Two outputs are available from the MZI, which are each injected into a separate core of the MCF. Fiber-optic circulators are used to direct the reflected signals from the gratings onto two photodetectors. A phase generated carrier (PGC) demodulation technique is used to extract the interferometric phase and hence the Bragg wavelengths of the FBG sensors $^{25}$. In this technique, a sinusoidal phase modulation of frequency $\omega_{p g c}(15 \mathrm{kHz})$ is applied to one of the arms of the MZI. It is assumed that the split ratios of the directional couplers in the MZI are 50\% and there is no excess loss in either interferometer arm. The current generated by the photodetector is then given by,

$$
i_{p h}=r P\left(1+V \cos \left[\phi_{p g c} \cos \omega_{p g c} t+\phi_{s}(t)+\phi_{d}(t)\right]\right)
$$

where $r$ is the photodiode responsivity, $P$ is the peak return power in the absence of the interference term, $V$ is the normalized fringe visibility, $\phi_{p g c}$ is the modulation depth, and $\phi_{s}(t)$ and $\phi_{d}(t)$ are signal and drift phases respectively. We set $\phi(t)=\phi_{s}(t)+\phi_{d}(t)$. Expanding the cosine term in (2) in terms of Bessel coefficients yields,

$$
i_{p h}=r P+r P V\left[\left[J_{o}\left(\phi_{p g c}\right)+2 \sum_{k=1}^{\infty}(-1)^{k} J_{2 k}\left(\phi_{p g c}\right) \cos 2 k \omega_{p g c} t\right] \cos \phi(t)-\left[2 \sum_{k=0}^{\infty}(-1)^{k} J_{2 k+1}\left(\phi_{p g c}\right) \cos \left((2 k+1) \omega_{p g c} t\right)\right] \sin \phi(t)\right]
$$

Thus, quadrature components of the phase of interest $\phi(t)$, can be obtained by synchronous detection of the photodiode current at $\omega_{p g c}$ and $2 \omega_{p g c}$. Low pass filtering the resulting signals yields,

$$
r P V J_{1}\left(\phi_{p g c}\right) \sin \phi(t)
$$




$$
-r P V J_{2}\left(\phi_{p g c}\right) \cos \phi(t) .
$$

Two lock-in amplifiers (LIA) are used to synchronously detect the photodiode current at $\omega_{p g c}$ and $2 \omega_{p g c}$ with a $90^{\circ}$ phase shift between them. These quadrature components are sampled using an analog-to-digital converter (A/D) and the phase of interest, $\phi(t)$, is calculated normalizing the amplitudes of (4) and (5) and taking the arctangent of their ratio. Setting $\phi_{p g c}$ equal to 2.6 radians results in $J_{1} \approx J_{2}$; however, a suitable normalization routine is also applied in the signal processing to remove any small differences in amplitude. Phase excursions greater than pi radians can be measured by implementing a fringe counting algorithm.

The phase is linearly related to the strain applied to the FBG, $\Delta \varepsilon$, by,

$$
\Delta \phi=\frac{2 \pi n L}{\lambda_{F B G}} 0.78 \Delta \varepsilon
$$

where $n$ is the fiber effective refractive index, $L$ is the interferometer fiber path imbalance, $\lambda_{F B G}$ is the Bragg wavelength of the FBG and the factor of 0.78 includes the contribution to the Bragg wavelength shift from the stressoptic effect ${ }^{26}$. In the experimental system, $n=1.468$ and $L=3.6 \mathrm{~mm} \pm 0.2 \mathrm{~mm}$ yielding, $\Delta \phi / \Delta \varepsilon=16.7 \mathrm{mrad} / \mu \varepsilon$.

\section{RESULTS}

\subsection{Quasi-static multicore fiber curvature sensor}

For this research, the differential strain between gratings in two adjacent cores was investigated. The MCF was approximately orientated to produce a maximum differential strain when the cantilever was displaced in the y-direction. The bend sensitivity in the vertical and horizontal planes was measured separately. The cantilever beam was displaced in steps of $1 \mathrm{~mm}$ in the $\mathrm{Y}$ axis up to a maximum of $5 \mathrm{~mm}$ and then returned to the start point. Due to the decreased sensitivity in the horizontal plane steps of $2.5 \mathrm{~mm}$ were used up to a maximum of $5 \mathrm{~mm}$. The quadrature components for each core were continuously acquired. The change in phase for each core was calculated and the phase differences for the displacement in the $\mathrm{Y}$ and $\mathrm{X}$ axes are shown in Fig. 3 (a) and (b) respectively. Since the Bragg wavelengths are not identical in the separate cores of the MCF, a correction factor must be included due to the wavelength dependence of the phase shift in (6), before the differential strain is calculated. The phase difference at each displacement step is plotted versus the applied displacement for the $\mathrm{Y}$ and $\mathrm{X}$ axes in Fig. 3 (c) and (d) respectively; linear polynomial fits were applied to the differential phase versus displacement. The azimuthal orientation of the sensor was determined from the arctangent of the ratio of the phase sensitivity with displacement for bending in X $(8.34 \mathrm{rad} / \mathrm{m})$ to bending in $\mathrm{Y}(59.04$ $\mathrm{rad} / \mathrm{m}$ ) to be $8^{\circ}$. For the bending configuration used here this is equivalent to a phase sensitivity with respect to curvature of $0.7933 \mathrm{rad} / \mathrm{m}^{-1}$. This compares well with the theoretical value of $0.8268 \mathrm{rad} / \mathrm{m}^{-1}$, the $\sim 4 \%$ variation is within the calibration accuracy of the interrogating interferometer.

\subsection{Drift/Accuracy}

The DC drift of the sensor and interrogation system were recorded and are shown in Fig. 4. The measured phase for each grating are shown in Fig. 4 (a) and clearly show large variations, $\sim 30$ radians, as a function of time. The primary cause of these variations is the sensitivity of the MZI and optical fiber downlead to environmental perturbations; however perturbations in the MZI are common to the interrogation of both gratings. Thus the differential phase yields a much smaller variation. The drift in the measured differential strain has been calculated from the differential phase which was low-pass filtered using a running average of duration $1 \mathrm{sec}$ and is plotted in Fig. 4 (b). This plot clearly indicates a very high differential strain stability with an r.m.s. deviation of $0.05 \mu \varepsilon$, which is equivalent to a curvature of $1 \times 10^{-3} \mathrm{~m}^{-1}$. However, in sensing environments where the interrogating interferometer and sensor downlead are perturbed by environmental fluctuations, the measurement accuracy will be degraded. The accuracy of the interrogation system is affected by errors in the interferometric phase measurement and polarization induced phase measurement errors, which arise due to a combination of the degree of polarization of the broadband source, birefringence in the interrogating interferometer and birefringence in the downlead and in the FBGs. A more detailed analysis of these effects are reported elsewhere ${ }^{27}$; however to achieve the lowest possible polarization induced phase errors it is important to minimize the birefringence in the system and illuminate it with unpolarized light. 


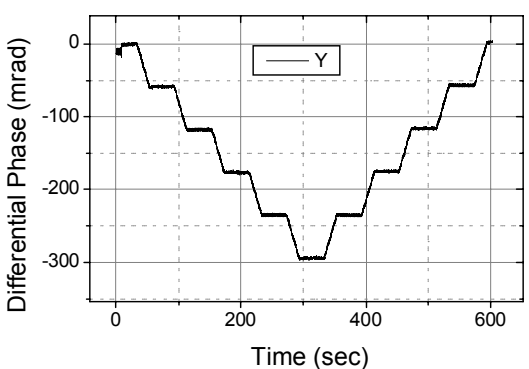

(a)

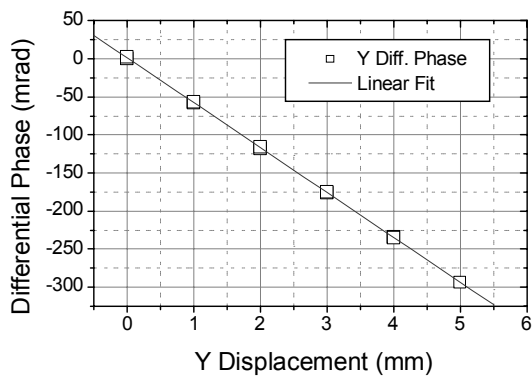

(c)

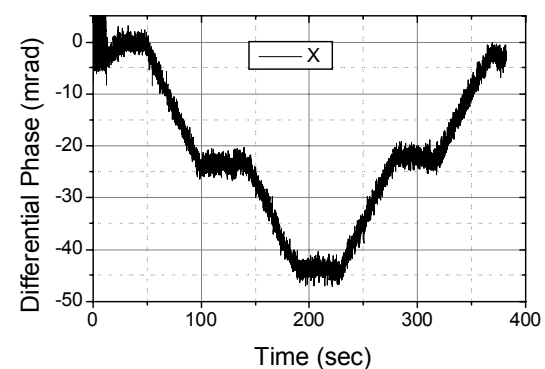

(b)

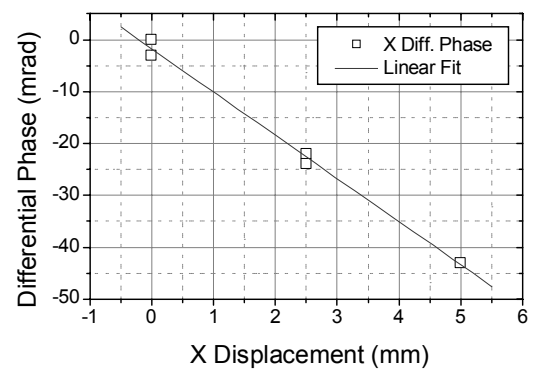

(d)

Fig. 3. (a) and (b) Measured phase difference versus time for displacement in Y-axis and X-axis respectively, (c) and (d) phase difference versus applied displacement in $\mathrm{Y}$-axis and $\mathrm{X}$-axis respectively with linear fits.

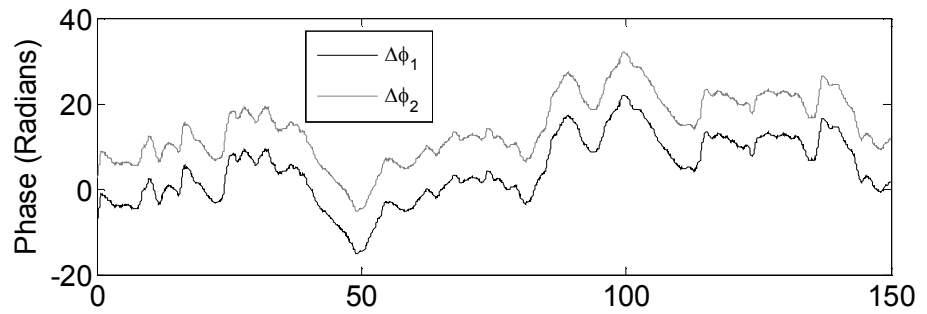

(a)

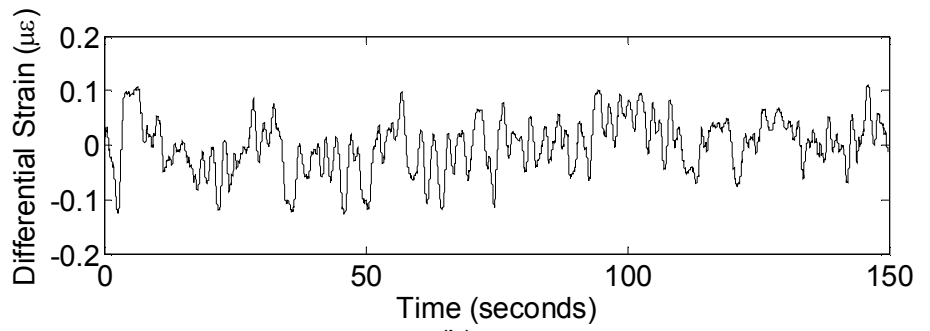

(b)

Fig. 4. (a) Phase drift of MCF sensor and (b) differential strain noise. 


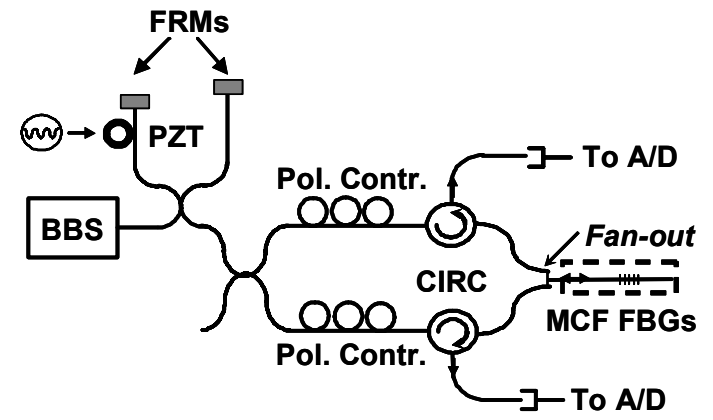

Fig. 5 Interrogation system using Michelson interferometer

In a practical system we can minimize the birefringence of the interrogating Mach-Zehnder interferometer by replacing it with a fiber Michelson interferometer with Faraday rotator mirrors ${ }^{28}$. The Michelson interferometer had a total round-trip fiber path imbalance of $L=6.4 \mathrm{~mm}(2 \times 3.2 \mathrm{~mm}$ arm imbalance). The phase to strain sensitivity for the Michelson interferometer is calculated to be $\Delta \phi / \Delta \varepsilon=29.7 \mathrm{mrad} / \mu \varepsilon$. The degree of polarization of the erbium broadband source was measured to be less than $3 \%$. To investigate the accuracy of the interrogation system we have placed two polarization controllers, one in each connecting fiber, between the output of the Michelson interferometer and the MCF FBGs. The birefringence in the fiber downleads can be arbitrarily varied by adjusting the polarization controllers during the measurement to determine the magnitude of the phase error due to downlead perturbations.

Birefringence in the FBG structures was also characterized by measuring the insertion loss of each grating as a function of polarization using a polarization sensitive optical frequency domain reflectometer, (OVA-CT, Luna Technologies, Blacksburg, VA). The reflection spectra for illumination by orthogonal polarization states for cores 2 and 3 are shown in Fig. 6 (a) and (b) respectively. Core 2 exhibits a large wavelength variation in comparison to core 3 for orthogonal polarization states. The wavelength of each FBG spectra were calculated using a centroid algorithm and the wavelength separation between orthogonal polarization states were calculated and are shown in Table 1. The refractive index difference $\Delta n$ was calculated using $\Delta n=\Delta \lambda / \Lambda_{P M}$, where $\Delta \lambda$ is the Bragg wavelength separation and $\Lambda_{P M}$ is the pitch of the phase-mask used to fabricate the gratings.

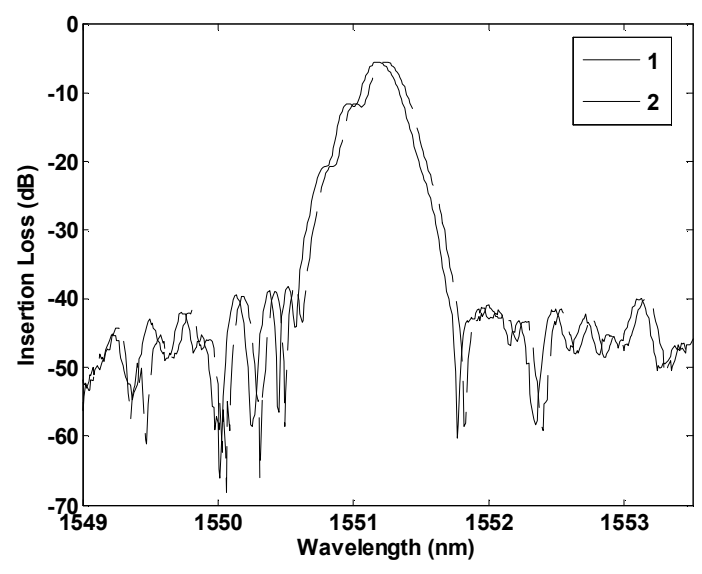

(a)

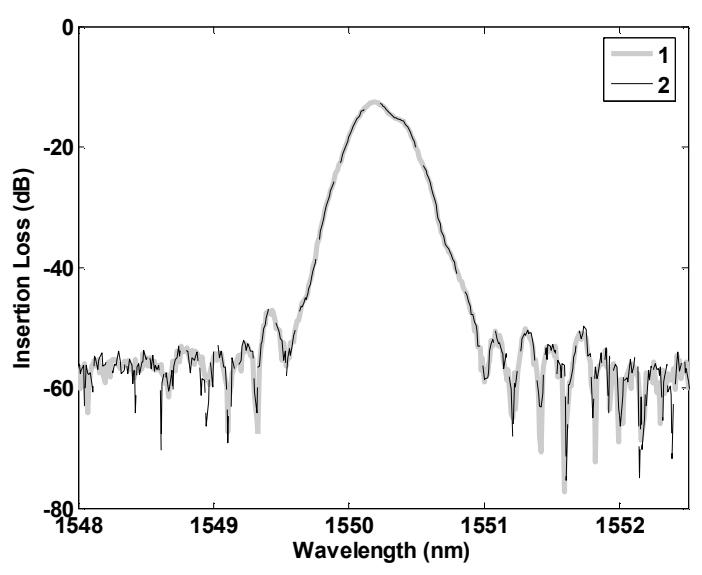

(b)

Fig. 6. Reflection spectra corresponding to the eigenaxes of the MCF FBG in core 2 (a) and core 3 (b) 


\begin{tabular}{|c|c|c|}
\hline Core & $\Delta \lambda(\mathrm{pm})$ & $\Delta \mathrm{n}$ \\
\hline 1 & 36.6 & $3.42 \times 10^{-5}$ \\
\hline 2 & 53.2 & $4.97 \times 10^{-5}$ \\
\hline 3 & 1.8 & $1.72 \times 10^{-6}$ \\
\hline 4 & 8.8 & $8.22 \times 10^{-6}$ \\
\hline
\end{tabular}

Table 1. Birefringence of FBGs in MCF

The polarization induced phase errors were measured by manually adjusting the polarization controller in a random fashion for one core at a time. Fig 7. shows the measured phase responses for cores 1 and 2 and also the differential phase between these cores, a low-pass filter running average of duration $0.1 \mathrm{sec}$ has been applied to all traces. The resultant phase error can be clearly seen in the each phase measurement and the differential phase response. The magnitude of this error can be observed to be approximately $\pm 15 \mathrm{mrad}$ for core 1 and $\pm 40 \mathrm{mrad}$ for core 2 . The increased phase error observed for core 2 is due to the larger birefringence in this core. The phase errors for cores 3 and 4 have also been measured and are $\pm 4 \mathrm{mrad}$ and $\pm 5 \mathrm{mrad}$ respectively.

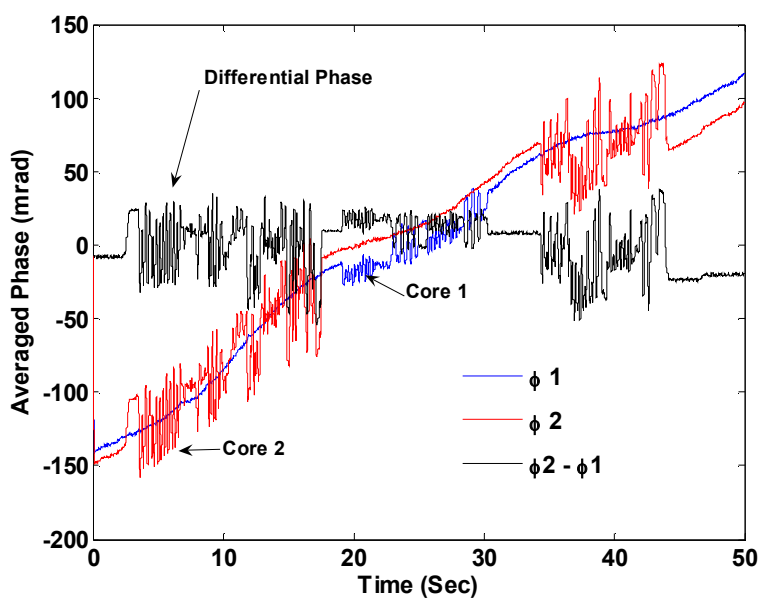

(a)

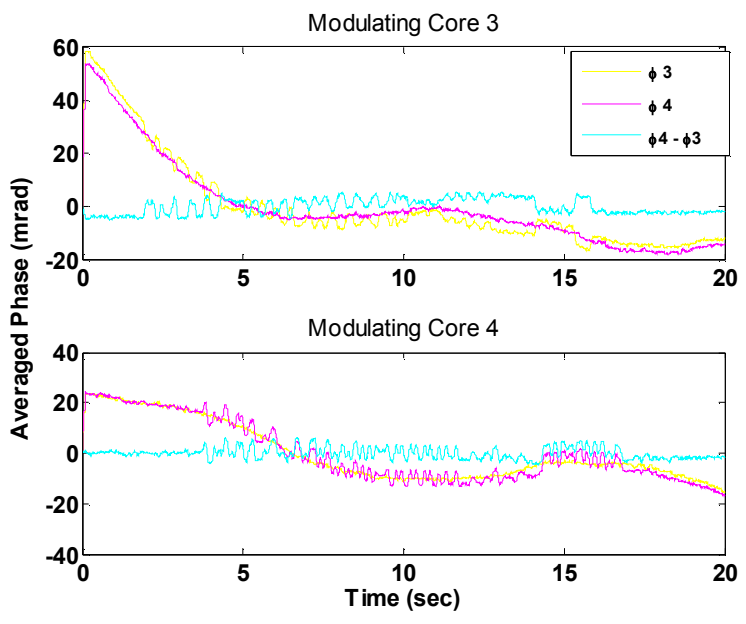

(b)

Fig. 7 Phase errors as a result of polarization modulation in the optical fiber downleads to cores 1 and 2 of the MCF (a) and to cores 3 and 4 (b).

By illuminating the optical system with an unpolarized source, i.e. a source whose degree of polarization is measured to be zero over the observation time, birefringence fluctuations in the interferometer, downlead and FBG do not directly induce phase measurement errors, provided there is no polarization dependent loss (PDL) in the optical system. Optical components with PDL will change the degree of polarization; therefore any polarization induced phase errors will depend on the changing DOP and birefringence throughout the optical system.

\subsection{Dynamic curvature sensing}

Dynamic curvature measurements have also been demonstrated by applying a displacement to the cantilever beam with a piezoelectric actuator with a $5 \mathrm{~Hz}$ modulation frequency. Fig. 8 shows the power spectrum of the measured phase for each core and the calculated differential phase. The signal at $5 \mathrm{~Hz}$ is clearly evident in all three signals; the inset details the difference in magnitude at $5 \mathrm{~Hz}$. Also, note that other low frequency effects above and below $5 \mathrm{~Hz}$ are also clearly cancelled. The power spectrum of the differential strain is shown in Fig. 9; the AC strain resolution is approximately $6 \mathrm{n} \varepsilon / \mathrm{Hz}^{1 / 2}$, which is equivalent to a curvature resolution of $1.2 \times 10^{-4} \mathrm{~m}^{-1}$. 


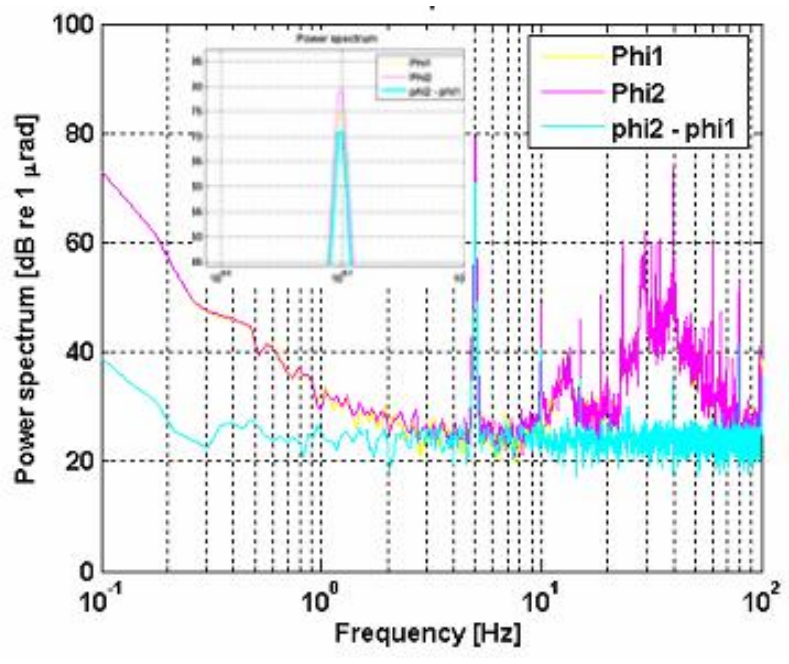

Fig. 8. Sensor response to $5 \mathrm{~Hz}$ applied curvature.

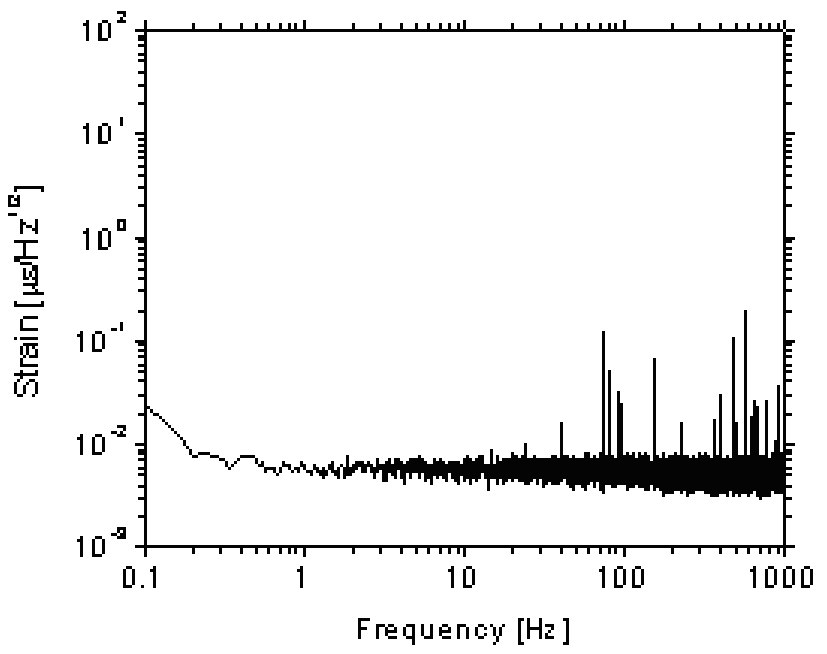

Fig. 9. AC differential strain resolution.

\section{DISCUSSION AND CONCLUSIONS}

We have applied a differential interferometric demodulation technique to differential strain sensing in a multicore optical fiber. By using a common interferometer as a wavelength discriminator this approach allows us to realize high resolution quasi-static and dynamic curvature measurements. A DC curvature accuracy of $3.0 \times 10^{-3} \mathrm{~m}^{-1}$, and an AC curvature resolution of $1.2 \times 10^{-4} \mathrm{~m}^{-1}$ have been demonstrated. To demonstrate the suitability of the interrogation technique for differential strain sensing, we have only interrogated two cores of the MCF; however a simple coupler tree would allow all cores to be interrogated simultaneously. The current interrogation system uses two lock-in-amplifiers per channel; however it is possible to implement the PGC demodulation technique in software after direct sampling of the interferometer outputs. Efficient multiplexing of FBGs using this interrogation technique has been investigated ${ }^{29}$, and shows the potential to multiplex up to 500 FBGs in each core.

\section{ACKNOWLEDGEMENTS}

The authors would like to thank Gary A. Fleming of the National Aeronautics and Space Administration, Langley Research Center, for provision of the multicore optical fiber used in this work.

\section{REFERENCES}

1. R. M. Measures, "Advances toward Fiber Optic Based Smart Structures," Optical Engineering, 31, pp. 34-47, 1992.

2. $\quad$ A. L. Window and G. S. Holister, Strain Gauge Technology: Applied Science Publishers, 1982.

3. A. D. Kersey, M. A. Davis, H. J. Patrick, M. LeBlanc, K. P. Koo, C. G. Askins, M. A. Putnam, and E. J. Friebele, "Fiber grating sensors," Journal of Lightwave Technology, 15, pp. 1442-1463, 1997.

4. $\quad$ Y. J. Rao, "Recent progress in applications of in-fibre Bragg grating sensors," Optics and Lasers in Engineering, 31, pp. 297-324, 1999.

5. R. T. Jones, D. G. Bellemore, T. A. Berkoff, J. S. Sirkis, M. A. Davis, M. A. Putnam, E. J. Friebele, and A. D. Kersey, "Determination of cantilever plate shapes using wavelength division multiplexed fiber Bragg grating sensors and a least-squares strain-fitting algorithm," Smart Materials \& Structures, 7, pp. 178-188, 1998.

6. M. G. Xu, J. L. Archambault, L. Reekie, and J. P. Dakin, "Thermally-Compensated Bending Gauge Using Surface-Mounted Fiber Gratings," International Journal of Optoelectronics, 9, pp. 281-283, 1994.

7. A. Djordjevich and M. Boskovic, "Curvature gauge," Sensors and Actuators A (Physical), A51, pp. 193-198, 1995.

8. L. A. Danisch, "Fiber-optic shape sensors (TM) and shape tape (TM)," Measurements \& Control, pp. 99-102, 1997. 
9. $\quad$ H. J. Patrick, C. C. Chang, and S. T. Vohra, "Long period fibre gratings for structural bend sensing," Electronics Letters, 34, pp. 1773-1775, 1998.

10. C. C. Ye, S. W. James, and R. P. Tatam, "Simultaneous temperature and bend sensing with long-period fiber gratings," Optics Letters, 25, pp. 1007-1009, 2000.

11. Y. Liu, L. Zhang, J. A. R. Williams, and I. Bennion, "Optical bend sensor based on measurement of resonance mode splitting of long-period fiber grating," IEEE Photonics Technology Letters, 12, pp. 531-533, 2000.

12. H. J. Patrick, "Self-aligning, bipolar bend transducer based on long period grating written in eccentric core fibre," Electronics Letters, 36, pp. 1763-1764, 2000.

13. T. Allsop, A. Gillooly, V. Mezentsev, T. Earthgrowl-Gould, R. Neal, D. J. Webb, and I. Bennion, "Bending and orientational characteristics of long period gratings written in D-Shaped optical fiber," IEEE Transactions on Instrumentation and Measurement, 53, pp. 130-135, 2004.

14. D. H. Zhao, X. F. Chen, K. M. Zhou, L. Zhang, I. Bennion, W. N. MacPherson, J. S. Barton, and J. D. C. Jones, "Bend sensors with direction recognition based on long-period gratings written in D-shaped fiber," Applied Optics, 43, pp. 5425-5428, 2004.

15. D. H. Zhao, K. M. Zhou, X. F. Chen, L. Zhang, I. Bennion, G. Flockhart, W. N. MacPherson, J. S. Barton, and J. D. C. Jones, "Implementation of vectorial bend sensors using long-period gratings UV-inscribed in special shape fibres," Measurement Science \& Technology, 15, pp. 1647-1650, 2004.

16. T. Allsop, M. Dubov, A. Martinez, F. Floreani, I. Khrushchev, D. J. Webb, and I. Bennion, "Long period grating directional bend sensor based on asymmetric index modification of cladding," Electronics Letters, 41, pp. 59-60, 2005.

17. Y. G. Han, B. H. Lee, W. T. Han, U. C. Paek, and Y. Chung, "Fibre-optic sensing applications of a pair of longperiod fibre gratings," Measurement Science \& Technology, 12, pp. 778-781, 2001.

18. Y. P. Wang and Y. J. Rao, "A novel long period fiber grating sensor measuring curvature and determining bend-direction simultaneously," IEEE Sensors Journal, 5, pp. 839-843, 2005.

19. F. M. Araujo, L. A. Ferreira, J. L. Santos, and F. Farahi, "Temperature and strain insensitive bending measurements with D-type fibre Bragg gratings," Measurement Science \& Technology, 12, pp. 829-833, 2001.

20. F. M. Araujo, L. A. Ferreira, and J. L. Santos, "Simultaneous determination of curvature, plane of curvature, and temperature by use of a miniaturized sensing head based on fiber Bragg gratings," Applied Optics, 41, pp. 2401-2407, 2002.

21. G. M. H. Flockhart, W. N. MacPherson, J. S. Barton, J. D. C. Jones, L. Zhang, and I. Bennion, "Two-axis bend measurement with Bragg gratings in multicore optical fiber," Optics Letters, 28, pp. 387-389, 2003.

22. A. D. Kersey, T. A. Berkoff, and W. W. Morey, "Fiberoptic Bragg Grating Strain Sensor with DriftCompensated High-Resolution Interferometric Wavelength-Shift Detection," Optics Letters, 18, pp. 72-74, 1993.

23. G. A. Johnson, M. D. Todd, B. L. Althouse, and C. C. Chang, "Fiber Bragg grating interrogation and multiplexing with a 3 x 3 coupler and a scanning filter," Journal of Lightwave Technology, 18, pp. 1101-1105, 2000.

24. P. P. Benham, R. J. Crawford, and C. G. Armstrong, Mechanics of Engineering Materials. London: AddisonWesley Longman, 1996.

25. A. Dandridge, A. B. Tveten, and T. G. Giallorenzi, "Homodyne Demodulation Scheme for Fiber Optic Sensors Using Phase Generated Carrier," IEEE Journal of Quantum Electronics, 18, pp. 1647-1653, 1982.

26. C. D. Butter and G. B. Hocker, "Fiber Optics Strain-Gauge," Applied Optics, 17, pp. 2867-2869, 1978.

27. G. A. Cranch, G. M. H. Flockhart, and C. K. Kirkendall, "Polarization Properties of Interferometrically Interrogated Fiber Bragg Grating and Tandem Interferometer Strain Sensors," accepted for publication in Journal of Lightwave Technology.

28. M. A. Davis and A. D. Kersey, "Application of a Fiber Fourier-Transform Spectrometer to the Detection of Wavelength-Encoded Signals from Bragg Grating Sensors," Journal of Lightwave Technology, 13, pp. 12891295, 1995.

29. G. A. Cranch, G. M. H. Flockhart, and C. K. Kirkendall, "Efficient fiber Bragg grating and fiber Fabry-Perot sensor multiplexing scheme using a broadband pulsed mode-locked laser," Journal of Lightwave Technology, 23, pp. 3798-3807, 2005. 